\title{
Blow-up solutions appearing in the vorticity dynamics with linear strain
}

\author{
K.-I. Nakamura, H. Okamoto ${ }^{\dagger}$ and H. Yagisita ${ }^{\ddagger}$
}

November 12, 2002

\begin{abstract}
We consider a model equation for $3 \mathrm{D}$ vorticity dynamics of incompressible viscous fluid proposed by K. Ohkitani and the second author of the present paper. We prove that a solution blows up in finite time if the $L^{1}$-norm of the initial vorticity is greater than the viscosity.
\end{abstract}

Keywords: blow-up, vorticity, Navier-Stokes

Subject Classification: 76D03, 76D05

Running title: blow-up in the vorticity dynamics with linear strain

*Dept. of Computer Science, University of Electro-Communications, Chofu, 182-8585 Japan

${ }^{\dagger}$ Research Institute for Mathematical Sciences, Kyoto University, Kyoto 606-8502 Japan. Partially supported by Grant-in-Aid of JSPS \# 14204007

${ }_{\ddagger}^{\ddagger}$ Dept. of Math., Faculty of Sci. and Tech., Tokyo University of Science, 2641 Yamazaki, Noda, Chiba Pref., 278-8510 Japan 


\section{Introduction}

An evolution equation which was derived from the Navier-Stokes equations in [18] is considered. It is written as follows:

$$
\begin{aligned}
\omega_{t} & =\|\omega(t)\|_{\infty}\left(r \omega_{r}+2 \omega\right)+\nu \frac{1}{r}\left(r \omega_{r}\right)_{r} \quad(0 \leq r<\infty, 0<t), \\
\omega(0, r) & =\omega_{0}(r),
\end{aligned}
$$

where $\nu>0$ is the kinematic viscosity, $\omega$ is a function of $(t, r), \omega(t)$ denotes $r$-function $\omega(t, \cdot)$, and \|\|$_{\infty}$ denotes the $L^{\infty}$-norm. This is derived from the vorticity dynamics in a linear strain field with the assumption that the strain-rate is proportional to the $L^{\infty}$-norm of the vorticity. Actually the velocity field $\mathbf{u}$ of incompressible viscous fluid in question is given by

$$
\mathbf{u}=(-\zeta(t) x+u(t, x, y),-\zeta(t) y+v(t, x, y), 2 \zeta(t) z)
$$

where $(x, y, z)$ denotes a point in three dimensional space $\mathbb{R}^{3}, \zeta(t)=\|\omega(t)\|_{\infty}$, and

$$
\begin{aligned}
& u(t, x, y)=\frac{-1}{2 \pi} \int_{\mathbb{R}^{2}} \frac{(y-\eta)}{(x-\xi)^{2}+(y-\eta)^{2}} \omega\left(t, \sqrt{\xi^{2}+\eta^{2}}\right) d \xi d \eta \\
& v(t, x, y)=\frac{1}{2 \pi} \int_{\mathbb{R}^{2}} \frac{(x-\xi)}{(x-\xi)^{2}+(y-\eta)^{2}} \omega\left(t, \sqrt{\xi^{2}+\eta^{2}}\right) d \xi d \eta
\end{aligned}
$$

( Note that curl $\mathbf{u}=(0,0, \omega(t, r))$ with $r=\sqrt{x^{2}+y^{2}}$. ) Although the assumption that the strain-rate $\zeta(t)$ is proportional to $\|\omega(t)\|_{\infty}$ is somewhat artificial, the solutions of (1) still are exact solutions of the three-dimensional Navier-Stokes equations ([18]). Hence it may be interesting to supply a mathematical proof of blow-up in finite time. Note however that our solutions are unbounded in $\mathbb{R}^{3}$, whence the energy is infinite. In particular, blow-up proved in the present paper do not have relevance to the famous problem of the regularity of the solutions of the Navier-Stokes equations $([5,12,14])$. Nevertheless, our results remind us that the energy inequality or something to specify the largeness of the velocity at infinity is necessary for the regularity of solutions of the 3D Navier-Stokes equations. For other aspects and related topics, see [7, 16, 18].

It was proved in [18] that, if $\|\omega(t)\|_{\infty}$ is replaced in (1) by the $L^{p}$-norm $\|\omega(t)\|_{p}$ with finite $p$, the solutions exist globally in time. The paper could not clarify the dynamics when $L^{\infty}$-norm was employed as in (1), although it found a self-similar blow-up solution of (1) and strongly suggested the blow-up for general data. The purpose of the present paper is to prove this ( Theorem 2 below ).

Numerical evidence exists for blow-up of certain exact unbounded solutions of the Navier-Stokes equations ( $[8,9,17,19])$, while global existence of some exact solutions are proved in $[3,18,21]$. Also, there exist mathematical proofs of blow-up for solutions of the 3D Euler equations with infinite energy ( $[4,15,21])$. However, it seems to be worthy of notice that the following two things hold true simultaneously in the Navier-Stokes equations ( as is implied by Theorems 1 and $\mathbf{2}$ below ):

1. there exists a class of functions in which the unique existence local-in-time of the solution is guaranteed: 
2. there exists a proof that some solution blows up in finite time.

Remark 1. A few words on the claim 1 above would be helpful. We prove the uniqueness of the solution of the vorticity equation, which in our case is (1). The velocity vector fields is constructed by (3), which may well be called a generalized Biot-Savart law, since its right hand side is a sum of the usual Biot-Savart term and an unbounded, irrotational flow. Therefore we cannot specify explicitly a uniqueness class in terms of $\mathbf{u}$. The uniqueness class is a thin, linear manifold of the space of bounded vorticity fields.

The present paper consists of four sections and an appendix. We give in section 2 a remark about non-existence of comparison theorem. The blow-up theorem is stated and proved in section 3. Section 4 is devoted to comments on remaining cases. Finally a proof of the unique existence of the solution local-in-time is given in Appendix.

\section{Non-existence of comparison theorem}

Before entering details of the result, we would like to show that the equation (1) does not admit a comparison theorem. We recall that self-similar blow-up solutions, which are written as

$$
\omega(t, r)=\frac{1+4 \alpha \nu}{2(T-t)} \exp \left(-\frac{\alpha r^{2}}{T-t}\right),
$$

where $T>0$ and $\alpha>0$ are parameters, were found in [18]. If a comparison theorem were valid, then the existence of a self-similar blow-up solution and comparison theorem would imply that any initial function which was larger at $t=0$ than a self-similar blow-up solution also blowed up in finite time. Although there is a possibility that this argument is correct, this way of proof would be very difficult, since there exists an example which shows that comparison between two general solutions is not guaranteed. Accordingly we are forced to look for a different approach to the proof of blow-up.

In order to see the invalidity of the comparison theorem, we consider two smooth functions $f_{1}(r)$ and $f_{2}(r)$ satisfying the following conditions:

- $f_{1}(r) \geq f_{2}(r)>0$ for all $r \in[0, \infty)$,

- $\left\|f_{1}\right\|_{\infty}>\left\|f_{2}\right\|_{\infty}$

- there exists an $r_{0}>0$ such that, $f_{1}\left(r_{0}\right)=f_{2}\left(r_{0}\right), f_{1}^{\prime}\left(r_{0}\right)=f_{2}^{\prime}\left(r_{0}\right)$, and $r_{0} f_{1}^{\prime}\left(r_{0}\right)+$ $2 f_{1}\left(r_{0}\right)=r_{0} f_{2}^{\prime}\left(r_{0}\right)+2 f_{2}\left(r_{0}\right)<0$.

Then $w \equiv \omega_{1}(t, r)-\omega_{2}(t, r)$, where $\omega_{j}$ denotes the solution of (1) with $f_{j}$ as its initial data $(j=1,2)$, satisfies

$$
w_{t}\left(0, r_{0}\right)=\left(\left\|f_{1}\right\|_{\infty}-\left\|f_{2}\right\|_{\infty}\right)\left(2 r_{0} f_{1}^{\prime}\left(r_{0}\right)+2 f_{1}\left(r_{0}\right)\right)+\nu\left(f_{1}-f_{2}\right)^{\prime \prime}\left(r_{0}\right) .
$$

( This equation is valid because both $\omega_{1}$ and $\omega_{2}$ are continuous at $t=0$, see Theorem 1 below. ) The right hand side is negative if $\nu$ is small enough. Since $w\left(0, r_{0}\right)=0$, $w\left(t, r_{0}\right)$ is negative for small $t>0$, while $w$ is nonnegative everywhere at $t=0$. 


\section{$3 \quad$ Finite time blow-up}

We now prove that large initial data lead to blow-up in finite time. Before doing so, we present some facts, which hold for any solutions without an assumption of the largeness of the initial data.

Let \|\|$_{1}$ be defined by

$$
\|f\|_{1} \equiv \int_{0}^{\infty}|f(r)| r d r<\infty
$$

and let $X_{1}$ be the set of all bounded, uniformly continuous functions defined in $[0, \infty)$ such that $\|f\|_{1}<\infty$. With $\|f\|_{1}+\|f\|_{\infty}$ as its norm, $X_{1}$ is clearly a Banach space. Let $X_{2}$ be the set of all the function $f:[0, \infty) \rightarrow \mathbb{R}$ such that $f$ is continuous and bounded in $[0, \infty)$ and satisfies

$$
\|f\|_{*} \equiv \sup _{0 \leq r<\infty} r|f(r)|<\infty
$$

Equipped with $\|f\|_{\infty}+\|f\|_{*}, X_{2}$ is a Banach space. Note that any function in $X_{2}$ is uniformly continuous in $[0, \infty)$. We then have the following

Theorem 1 For all $\omega_{0} \in X_{1} \cap X_{2}$, there exists $T_{1}>0$ depending only on $\left\|\omega_{0}\right\|_{1}+$ $\left\|\omega_{0}\right\|_{\infty}+\left\|\omega_{0}\right\|_{*}$ such that the solution of $(1)$ and $(2)$ exists and unique in $C^{0}\left(\left[0, T_{1}\right] ; X_{1}\right) \cap$ $L^{\infty}\left(0, T_{1} ; X_{2}\right)$.

We postpone the proof until we outline it in Appendix, since the local existence is not a central issue and its proof is carried out by standard arguments. The reader, however, may wonder if the function space $X_{1} \cap X_{2}$ is the largest one for the unique existence. In particular, one may wonder if the somewhat artificial condition $\sup r|f(r)|<\infty$ can be weakened. We do not know the answer. Since this function space suits our physical motivation in which the vortices are localized near the $z$-axis ( [18] ), we would not try to find an optimal result.

Throughout the remaining part of the present paper, we consider those solutions with $\omega_{0}(r) \geq 0$ everywhere. Despite the failure of the comparison theorem, we can still prove that $\omega(t, r) \geq 0$ for all $t$ and $r$. We can also prove easily that the $L^{1}$-norm of $\omega(t)$ is conserved by the evolution equation (1). Accordingly, we have $\|\omega(t)\|_{1}=\int_{0}^{\infty} \omega(t, r) r d r=$ $\left\|\omega_{0}\right\|_{1}$ for all $t \geq 0$. Therefore, the global existence is guaranteed if the a priori estimate $\sup _{0<t<T}\|\omega(t)\|_{\infty}<\infty$ and $\sup _{0<t<T}\|\omega(t)\|_{*}<\infty$ hold for any $T>0$.

Let $[0, T)$ be the maximum interval of the existence of the solution $\omega(t) .(T=\infty$ is included. ) We define two functions $a$ and $b$ of $t \in[0, T)$ by

$$
a(t)=\exp \left(\int_{0}^{t}\|\omega(s)\|_{\infty} d s\right), \quad b(t)=\int_{0}^{t} a(s)^{2} d s .
$$

They are increasing functions and satisfy $a(t) \geq 1$ and $b(t) \geq t$ in $0 \leq t<T$. We next define $u=u(\tau, \xi)$ by

$$
\frac{\partial u}{\partial \tau}=\nu \frac{1}{\xi} \frac{\partial}{\partial \xi}\left(\xi \frac{\partial u}{\partial \xi}\right), \quad u(0, \xi)=\omega_{0}(\xi)
$$


or we may write as

$$
u(\tau, \xi)=\frac{1}{4 \pi \nu \tau} \int_{\mathbb{R}^{2}} \exp \left(-\frac{|\boldsymbol{\xi}-\mathbf{y}|^{2}}{4 \nu \tau}\right) \omega_{0}(|\mathbf{y}|) d y_{1} d y_{2},
$$

where $\boldsymbol{\xi}=(\xi, 0)$ and $\mathbf{y}=\left(y_{1}, y_{2}\right)$. Then it holds that

$$
\omega(t, r)=a(t)^{2} u(b(t), a(t) r) .
$$

( This nice trick is due to Lundgren [13]. )

Defining $h$ as $h(\tau)=2 \tau\|u(\tau)\|_{\infty}$, we can easily see that $\lim _{\tau \rightarrow 0} h(\tau)=0$, that $h$ is a monotone increasing function, and that we have

$$
\lim _{\tau \rightarrow \infty} h(\tau)=\frac{\left\|\omega_{0}\right\|_{1}}{\nu}
$$

In view of this, we define $\gamma=\left\|\omega_{0}\right\|_{1} / \nu$. Note also that we have the following differential equations

$$
\begin{aligned}
\dot{a} & =\frac{h(b)}{2 b} a^{3}, \\
\dot{b} & =a^{2}
\end{aligned}
$$

with initial data $a(0)=1$ and $b(0)=0$. From these equations, we have

$$
\frac{\dot{a}}{a}=\frac{1}{2} h(b) \frac{\dot{b}}{b} .
$$

Now take any $t_{0} \in(0, T)$ and fix it. Define $\tilde{\gamma}=h\left(b\left(t_{0}\right)\right)$. Then (11) and the monotonicity of $h$ yield

$$
\frac{\tilde{\gamma}}{2} \frac{\dot{b}}{b} \leq \frac{\dot{a}}{a} \leq \frac{\gamma}{2} \frac{\dot{b}}{b} \quad\left(t_{0} \leq t<T\right)
$$

By integration we obtain

$$
\left(\frac{b(t)}{b\left(t_{0}\right)}\right)^{\tilde{\gamma} / 2} \leq \frac{a(t)}{a\left(t_{0}\right)} \leq\left(\frac{b(t)}{b\left(t_{0}\right)}\right)^{\gamma / 2}
$$

These inequalities imply that

$$
\lim _{t \rightarrow T} a(t)=\lim _{t \rightarrow T} b(t)=\infty,
$$

in either case of $T<\infty$ or $T=\infty$ : in fact, if $T<\infty$, then we must have either $\lim _{t \rightarrow T}\|\omega(t)\|_{\infty}=\infty$ or $\lim _{t \rightarrow T}\|\omega(t)\|_{*}=\infty$ because of the local existence of the solution in $X_{1} \cap X_{2}$ and the invariance of the $L^{1}$-norm. Note that

$$
\begin{aligned}
\xi u(\tau, \xi)= & \frac{1}{4 \pi \nu \tau} \int \exp \left(-\frac{\left(\xi-y_{1}\right)^{2}+y_{2}^{2}}{4 \nu \tau}\right)\left(\xi-y_{1}\right) \omega_{0}(|y|) d y_{1} d y_{2} \\
& +\frac{1}{4 \pi \nu \tau} \int \exp \left(-\frac{\left(\xi-y_{1}\right)^{2}+y_{2}^{2}}{4 \nu \tau}\right) y_{1} \omega_{0}(|y|) d y_{1} d y_{2} \\
\leq & c \tau^{1 / 2}\left\|\omega_{0}\right\|_{\infty}+\left\|\omega_{0}\right\|_{*},
\end{aligned}
$$


so we have

$$
\|u(\tau)\|_{*} \leq c \tau^{1 / 2}\left\|\omega_{0}\right\|_{\infty}+\left\|\omega_{0}\right\|_{*},
$$

where $c$ is a constant. Hereafter we follow the usual convention that $c$ denotes a positive constant which may differ in different contexts. Note also that $\|u(\tau)\|_{\infty} \leq\left\|\omega_{0}\right\|_{\infty}$. Since

$$
\|\omega(t)\|_{\infty}=a(t)^{2}\|u(b(t))\|_{\infty}, \quad\|\omega(t)\|_{*}=a(t)\|u(b(t))\|_{*},
$$

either $a$ or $b$ must be unbounded, hence both are unbounded because of (12). If $T=\infty$, then $b(t) \geq t$, as is noted above, shows the unboundedness of $b$, and (12) implies (13).

We finally define $d$ by $d(t)=a(t)^{-2} b(t)$. It satisfies

$$
\dot{d}=1-h(b) \quad(0 \leq t<T) .
$$

The following lemma, which will play a crucial role later, is easily verified:

Lemma $1 d(t)$ is positive for all $t \in(0, T)$. $\dot{d}$ is monotone decreasing. It satisfies

$$
\|\omega(t)\|_{\infty}=\frac{h(b(t))}{2 d(t)} .
$$

We are now ready to prove the following theorem:

Theorem 2 Let $\omega_{0} \in X_{1} \cap X_{2}$ be non-negative in $[0, \infty)$. Then the solution blows up in finite time if $\left\|\omega_{0}\right\|_{1}>\nu$. Further, we have

$$
\lim _{t \rightarrow T}(T-t)\|\omega(t)\|_{\infty}=\frac{\gamma}{2(\gamma-1)},
$$

where $T$ is the blow-up time and $\gamma=\left\|\omega_{0}\right\|_{1} / \nu$.

Proof. Suppose that the solution exists for all $t \in[0, \infty)$. Then, since the right hand side of (15) tends to $1-\gamma<0, d(t)$ becomes negative for sufficiently large $t$. This is an obvious contradiction because $d$ is positive. $d(t)$ therefore exists only for finite time.

In order to prove (17), we take $t_{0}$ such that $\tilde{\gamma}=h\left(b\left(t_{0}\right)\right)>1$. This is possible if $t_{0}$ is sufficiently close to $T$ ( see (8) ). Then (12) and (13) imply that $\lim _{t \rightarrow T} d(t)=0$. This, together with (15), yields that

$$
\frac{d(t)}{T-t}=\frac{1}{T-t} \int_{t}^{T} h(b(s)) d s-1 \rightarrow h(b(T-0))-1=\gamma-1
$$

as $t \rightarrow T$. Consequently, by Lemma 1 , we have

$$
\lim _{t \rightarrow T}(T-t)\|\omega(t)\|_{\infty}=\lim _{t \rightarrow T} \frac{h(b(t))}{2} \frac{T-t}{d(t)}=\frac{\gamma}{2(\gamma-1)},
$$

and we are done.

Remark 2. It is interesting to note the self-similar blow-up solutions (4) satisfy

$$
\|\omega(0)\|_{1}=\nu+\frac{1}{4 \alpha}>\nu .
$$


Remark 3. The blow-up rate (17) complies with the famous criterion by Beale, Kato and Majda[1], although their theorem gives a blow-up criterion for the Euler equations, not for the Navier-Stokes equations. It also complies with Kozono and Taniuchi's blow-up criterion on the Navier-Stokes equations in [11].

Remark 4. Also important is to note that blow-up occurs only at $r=0$ if the initial function is monotone decreasing in $r$ and decays sufficiently rapidly as $r \rightarrow \infty$. Since the numerical solutions discovered by $[8,9,17]$ seem to blow up on the whole interval of space variable, not on a single point, the model (1) may be said to be potentially more relevant ( to real flow dynamics ) than those equations in $[8,9,17]$ are. However, the fact that the set of singular points is exactly the $z$-axis does not comply with the existence of a weak solution such that the one-dimensional Hausdorff measure of the ( possible ) singular set is zero ( $[2]$ ). This discrepancy is caused, of course, by the non-existence of the energy inequality.

\section{The case where $\left\|\omega_{0}\right\|_{1} \leq \nu$}

We here prove

Theorem 3 Assume that $\omega_{0} \in X_{1} \cap X_{2}$ is non-negative. If $\left\|\omega_{0}\right\|_{1} \leq \nu$, the solution of (1) and (2) exists for all $0 \leq t<\infty$. Further, $\sup _{0<t<\infty}\|\omega(t)\|_{\infty}<\infty$. If $\left\|\omega_{0}\right\|_{1}<\nu$, then

$$
\lim _{t \rightarrow \infty} t\|\omega(t)\|_{\infty}=\frac{\gamma}{2(1-\gamma)}
$$

In particular $\|\omega(t)\|_{\infty}$ tends to zero. If $\left\|\omega_{0}\right\|_{1}=\nu$, we have

$$
\liminf _{t \rightarrow \infty}\|\omega(t)\|_{\infty}>0
$$

provided that there exists a positive constant $\delta$ such that

$$
\int_{0}^{\infty} \omega_{0}(r) r^{1+\delta} d r<\infty
$$

Proof. By (12) we have

$$
\dot{b}=a^{2} \leq \frac{a\left(t_{0}\right)^{2}}{b\left(t_{0}\right)^{\gamma}} b(t)^{\gamma} \equiv M b(t)^{\gamma}
$$

for all $0<t_{0}<t$. Accordingly, we have

$$
\frac{b(t)^{1-\gamma}}{1-\gamma} \leq \frac{b\left(t_{0}\right)^{1-\gamma}}{1-\gamma}+M\left(t-t_{0}\right) \quad(\text { for } \gamma<1)
$$

or

$$
b(t) \leq b\left(t_{0}\right) \exp \left(M\left(t-t_{0}\right)\right) \quad(\text { for } \gamma=1)
$$

which implies that $b$ is locally bounded. The boundedness of $a$ follows from this and (12). Since $\|\omega(t)\|_{\infty}=a(t)^{2}\|u(b(t))\|_{\infty} \leq a(t)^{2}\left\|\omega_{0}\right\|_{\infty}$, the local boundedness of $\|\omega(t)\|_{\infty}$ follows. Also (14) implies that

$$
|r \omega(t, r)|=a(t)^{2} r|u(b(t), a(t) r)| \leq a(t)\|u(b(t))\|_{*} \leq c a(t)\left(b(t)^{1 / 2}\left\|\omega_{0}\right\|_{\infty}+\left\|\omega_{0}\right\|_{*}\right) .
$$


Therefore $\omega(t)$ is bounded in $X_{2}$ locally in time, and the possibility of $T<\infty$ is excluded.

By Lemma $1, \dot{d}(t) \geq 1-\gamma \geq 0$, whence $d(t) \geq d\left(t_{0}\right)>0$ for all $t \geq t_{0}$. We therefore have the following upper bound:

$$
\|\omega(t)\|_{\infty}=\frac{h(b(t))}{2 d(t)} \leq \frac{\gamma}{2 d\left(t_{0}\right)} .
$$

If $\gamma<1$, then Lemma 1 implies

$$
\lim _{t \rightarrow \infty} \frac{d(t)}{t}=\lim _{t \rightarrow \infty} \dot{d}(t)=1-\gamma .
$$

Accordingly

$$
t\|\omega(t)\|_{\infty}=\frac{h(b(t))}{2} \frac{t}{d(t)} \rightarrow \frac{\gamma}{2(1-\gamma)}
$$

as $t \rightarrow \infty$.

Finally, we note that, if $\gamma=1$,

$$
\begin{aligned}
\dot{d}(t) & \leq 1-\frac{1}{2 \pi \nu} \int_{\mathbb{R}^{2}} \exp \left(-\frac{|\mathbf{y}|^{2}}{4 \nu b(t)}\right) \omega_{0}(|\mathbf{y}|) d y_{1} d y_{2} \\
& =\frac{1}{\nu} \int_{0}^{\infty}\left[1-\exp \left(-\frac{r^{2}}{4 \nu b(t)}\right)\right] \omega_{0}(r) r d r \\
& \leq c b(t)^{-\delta / 2}
\end{aligned}
$$

On the other hand, (10) and (12) give us

$$
\dot{b} \geq k b^{\tilde{\gamma}} \quad\left(t_{0} \leq t\right)
$$

with a positive constant $k$ and $\tilde{\gamma}=h\left(b\left(t_{0}\right)\right)$. Hence

$$
b(t) \geq\left\{b\left(t_{0}\right)^{1-\tilde{\gamma}}+k(1-\tilde{\gamma})\left(t-t_{0}\right)\right\}^{1 /(1-\tilde{\gamma})}
$$

for $t \geq t_{0}$. Note that $\tilde{\gamma}=h\left(b\left(t_{0}\right)\right)$ can be chosen as closely to unity as we wish. If $1-\tilde{\gamma}<\delta / 2$, then

$$
d(t) \leq d\left(t_{0}\right)+c \int_{t_{0}}^{\infty} \frac{d s}{b(s)^{\delta / 2}} \equiv K<\infty .
$$

This implies

$$
\|\omega(t)\|_{\infty}=\frac{h(b(t))}{2 d(t)} \geq \frac{\tilde{\gamma}}{2 K} .
$$

Remark 5. There exist ( [18] ) the following steady-states:

$$
\omega_{0}(r)=2 \alpha \nu e^{-\alpha r^{2}},
$$

where $\alpha \in(0, \infty)$ is a parameter. Whatever $\alpha$ may be, we have $\left\|\omega_{0}\right\|_{1}=\nu$. 


\section{Appendix}

Proof of Theorem 1: Suppose that $\omega_{0} \in X_{1} \cap X_{2}$ is given. In this section, its sign does not matter. We now define

$$
\mathbf{Y}=\left\{A \in C^{0}([0, T]) ; 0 \leq A(t) \leq 2\left\|\omega_{0}\right\|_{\infty} \quad(t \in[0, T])\right\}
$$

Given any $A \in \mathbf{Y}$ we define $\omega$ by

$$
\begin{aligned}
\omega_{t} & =A(t)\left(r \omega_{r}+2 \omega\right)+\nu \frac{1}{r}\left(r \omega_{r}\right)_{r} \quad(0 \leq r<\infty, 0<t), \\
\omega(0, r) & =\omega_{0}(r) .
\end{aligned}
$$

We then define $\Phi(A)(t)=\|\omega(t)\|_{\infty}$ for $0 \leq t \leq T$. Our goal is then to prove the existence of a fixed point of the mapping $\Phi$. We begin with the proof of the fact that there exists a $T>0$ such that $\Phi$ maps $\mathbf{Y}$ into itself.

For a given $A$, we define $a$ and $b$ by

$$
a(t)=\exp \left(\int_{0}^{t} A(s) d s\right), \quad b(t)=\int_{0}^{t} a(s)^{2} d s .
$$

Defining $u=u(\tau, \xi)$ by (6), we have $\omega(t, r)=a(t)^{2} u(b(t), a(t) r)$. Since $\omega_{0}$ is uniformly continuous, $\|u(\tau)\|_{\infty}$ is continuous on $[0, T]$, which implies that $\|\omega(t)\|_{\infty}=a(t)^{2}\|u(b(t))\|_{\infty}$ is continuous on $[0, T]$. Further, we obtain

$$
\|\omega(t)\|_{\infty}=a(t)^{2}\|u(b(t))\|_{\infty} \leq a(t)^{2}\left\|\omega_{0}\right\|_{\infty} .
$$

Therefore $\Phi(A) \in \mathbf{Y}$, if $\exp \left(4 T\left\|\omega_{0}\right\|_{\infty}\right) \leq 2$, which is satisfied for a sufficiently small $T$. We fix such a $T$.

We next show that $\Phi$ is a contraction mapping if $T$ is small. Suppose that two functions $A_{1}$ and $A_{2}$ are given and define $a_{i}, b_{i}$ by

$$
a_{i}(t)=\exp \left(\int_{0}^{t} A_{i}(s) d s\right), \quad b_{i}(t)=\int_{0}^{t} a_{i}(s)^{2} d s \quad(i=1,2) .
$$

We then define $\omega_{i}(t, r)=a_{i}(t)^{2} u\left(b_{i}(t), a_{i}(t) r\right) \quad(i=1,2)$. Let

$$
\begin{aligned}
\omega_{1}(t)-\omega_{2}(t)= & \left(a_{1}^{2}-a_{2}^{2}\right) u\left(b_{1}, a_{1} r\right)+a_{2}^{2}\left[u\left(b_{1}, a_{1} r\right)-u\left(b_{2}, a_{1} r\right)\right] \\
& \quad+a_{2}^{2}\left[u\left(b_{2}, a_{1} r\right)-u\left(b_{2}, a_{2} r\right)\right] \\
\equiv & I_{1}+I_{2}+I_{3} .
\end{aligned}
$$

The right hand side is estimated as follows. Note first that

$$
\left|a_{1}(t)-a_{2}(t)\right| \leq c \int_{0}^{t}\left|A_{1}(s)-A_{2}(s)\right| d s \leq c t \max _{0 \leq t \leq T}\left|A_{1}(t)-A_{2}(t)\right|
$$

and

$$
\left|b_{1}(t)-b_{2}(t)\right| \leq c \int_{0}^{t}(t-s)\left|A_{1}(s)-A_{2}(s)\right| d s \leq \frac{c t^{2}}{2} \max _{0 \leq t \leq T}\left|A_{1}(t)-A_{2}(t)\right| .
$$


We now have

$$
\left|I_{1}\right| \leq c t \max _{0 \leq t \leq T}\left|A_{1}(t)-A_{2}(t)\right|
$$

Since $b_{i}(t) \geq t$ implies that

$$
\begin{aligned}
& \sup _{0 \leq r<\infty}\left|u\left(b_{1}, a_{1} r\right)-u\left(b_{2}, a_{1} r\right)\right|=\sup _{0 \leq r<\infty}\left|\int_{0}^{1} \frac{d}{d s} u\left(b_{2}+s\left(b_{1}-b_{2}\right), a_{1} r\right) d s\right| \\
& \quad \leq \int_{0}^{1}\left\|\frac{\partial u}{\partial \tau}\left(b_{2}+s\left(b_{1}-b_{2}\right)\right)\right\|_{\infty} d s\left|b_{1}-b_{2}\right| \leq \frac{c\left|b_{1}-b_{2}\right|}{\min \left(b_{1}(t), b_{2}(t)\right)} \\
& \quad \leq c t^{-1}\left|b_{1}(t)-b_{2}(t)\right|,
\end{aligned}
$$

we have

$$
\left|I_{2}\right| \leq c t \max _{0 \leq t \leq T}\left|A_{1}(t)-A_{2}(t)\right| .
$$

The term $I_{3}$ is estimated as follows:

$$
\begin{aligned}
u\left(b_{2}, a_{1} r\right)-u\left(b_{2}, a_{2} r\right) & =\int_{0}^{1} \frac{d}{d s} u\left(b_{2}, a_{2} r+s r\left(a_{1}-a_{2}\right)\right) d s \\
& =\int_{0}^{1} u_{\xi}\left(b_{2}, a_{2} r+\operatorname{sr}\left(a_{1}-a_{2}\right)\right)\left(a_{1}-a_{2}\right) r d s
\end{aligned}
$$

Since $1 \leq \min \left(a_{1}, a_{2}\right) \leq a_{2}+s\left(a_{1}-a_{2}\right)$, we obtain

$$
\sup _{0 \leq r<\infty}\left|u\left(b_{2}, a_{1} r\right)-u\left(b_{2}, a_{2} r\right)\right| \leq \sup _{0 \leq \xi<\infty}\left|\xi u_{\xi}\left(b_{2}, \xi\right)\right|\left|a_{1}-a_{2}\right| .
$$

By a standard argument we have

$$
\begin{aligned}
\xi \frac{\partial u}{\partial \xi}(\tau, \xi)= & \frac{-1}{4 \pi \nu \tau} \int \exp \left(-\frac{\left(\xi-y_{1}\right)^{2}+y_{2}^{2}}{4 \nu \tau}\right) \frac{\xi\left(\xi-y_{1}\right)}{2 \nu \tau} \omega_{0}(|y|) d y_{1} d y_{2} \\
= & \frac{-1}{4 \pi \nu \tau} \int \exp \left(-\frac{\left(\xi-y_{1}\right)^{2}+y_{2}^{2}}{4 \nu \tau}\right) \frac{\left(\xi-y_{1}\right)^{2}}{2 \nu \tau} \omega_{0}(|y|) d y_{1} d y_{2} \\
& +\frac{-1}{4 \pi \nu \tau} \int \exp \left(-\frac{\left(\xi-y_{1}\right)^{2}+y_{2}^{2}}{4 \nu \tau}\right) \frac{y_{1}\left(\xi-y_{1}\right)}{2 \nu \tau} \omega_{0}(|y|) d y_{1} d y_{2} \\
\leq & \left\|\omega_{0}\right\|_{\infty} \frac{1}{4 \pi \nu \tau} \int \exp \left(-\frac{\left(\xi-y_{1}\right)^{2}+y_{2}^{2}}{4 \nu \tau}\right) \frac{\left(\xi-y_{1}\right)^{2}}{2 \nu \tau} d y_{1} d y_{2} \\
& +\left\|\omega_{0}\right\|_{*} \frac{1}{4 \pi \nu \tau} \int \exp \left(-\frac{\left(\xi-y_{1}\right)^{2}+y_{2}^{2}}{4 \nu \tau}\right)\left|\frac{\xi-y_{1}}{2 \nu \tau}\right| d y_{1} d y_{2} \\
\leq & c\left\|\omega_{0}\right\|_{\infty}+c \tau^{-1 / 2}\left\|\omega_{0}\right\|_{*} .
\end{aligned}
$$

This then yields

$$
\left|I_{3}\right| \leq c\left(1+t^{-1 / 2}\right)\left|a_{1}(t)-a_{2}(t)\right| \leq c\left(t+t^{1 / 2}\right) \max _{0 \leq t \leq T}\left|A_{1}(t)-A_{2}(t)\right| .
$$


Combining (19)-(21) with (18), we obtain

$$
\left|\omega_{1}(t)-\omega_{2}(t)\right| \leq c\left(t+t^{1 / 2}\right) \max _{0 \leq t \leq T}\left|A_{1}(t)-A_{2}(t)\right|
$$

Namely,

$$
\max _{0 \leq t \leq T}\left|\Phi\left(A_{1}\right)(t)-\Phi\left(A_{2}\right)(t)\right| \leq c\left(T+T^{1 / 2}\right) \max _{0 \leq t \leq T}\left|A_{1}(t)-A_{2}(t)\right| .
$$

Accordingly $\Phi$ is a contraction mapping for sufficiently small $T$, and it has a unique fixed point in $\mathbf{Y}$.

The $\omega(t, r)$ constructed by the fixed point is what we are looking for. Using the fact that $-\triangle$ generates an analytic semigroup in $L^{1}$ and in the space of bounded uniformly continuous functions, it is not difficult to verify that $\omega \in C^{0}\left([0, T] ; X_{1}\right) \cap L^{\infty}\left((0, T) ; X_{2}\right)$ ( cf. $[10,20]$ ).

As for uniqueness, we recall that any continuous and bounded solution $\omega$ can be represented as (7). Then, for sufficiently small $T>0,\|\omega(\cdot)\|_{\infty}$ belongs to $\mathbf{Y}$. The uniqueness in $0 \leq t<T$ is then a consequence of the contraction mapping theorem. Since this argument can be repeated, we get to the uniqueness.

\section{References}

[1] J.T. Beale, T. Kato, and A. Majda, Remarks on the breakdown of smooth solutions for the 3-D Euler equations, Comm. Math. Phys., 94 (1984), pp. 61-66.

[2] L. Caffarelli, R. Kohn, and L. Nirenberg, Partial regularity of suitable weak solutions of the Navier-Stokes equations, Comm. Pure Appl. Math., 35, 771-831 (1982)

[3] X. Chen and H. Okamoto, Global Existence of Solutions to the Proudman-Johnson Equation, Proc. Japan Acad., 76, 149-152 (2000).

[4] P. Constantin, The Euler equations and non-local conservative Riccati equations, Inter. Math. Res. Notice, (2000), No. 9, pp. 455-465.

[5] C. R. Doering and J. D. Gibbon, Applied Analysis of the Navier-Stokes Equations, Cambridge Univ. Press (1995).

[6] Y. Giga and M.-H. Giga, Nonlinear Partial Differential Equations, Kyoritsu Shuppan ( in Japanese ) (1999).

[7] Y. Giga and T. Kambe, Large time behavior of the vorticity of two-dimensional viscous flow and its application to vortex formation, Commun. Math. Phys., 117, 549-568 (1988).

[8] R. E. Grundy and R. McLaughlin, Global blow-up of separable solutions of the vorticity equation, IMA J. Appl. Math., 59, 287-307 (1997).

[9] R. E. Grundy and R. McLaughlin, Three-dimensional blow-up solutions of the NavierStokes equations, IMA J. Appl. Math., 63, 287-306 (1999). 
[10] T. Kato, Perturbation Theory for Linear Operators, 2nd Ed., Springer Verlag (1980).

[11] H. Kozono and Y. Taniuchi, Bilinear estimates in BMO and the Navier-Stokes equations, Math. Z., 235, 173-19 (2000).

[12] P.-L. Lions, Mathematical Topics in Fluid Mechanics, Vol. I, Incompressible Models, Clarendon Press, Oxford (1996).

[13] T. S. Lundgren, Strained spiral vortex model for turbulent fine structure, Phys. Fluid, $252193-2203$ (1982).

[14] A. J. Majda and A. L. Bertozzi, Vorticity and Incompressible Flow, Cambridge Univ. Press (2002),

[15] S. J. A. Malham, Collapse of a class of three-dimensional Euler vortices, Proc. R. Soc. Lond., A 456 (2000), pp. 2823-2833.

[16] H. K. Moffatt, The interaction of skewed vortex pairs: a model for blow-up of the Navier-Stokes equations, J. Fluid Mech., 409, 51-68 (2000).

[17] M. Nagayama, H. Okamoto, J. Zhu, On the blow-up of some similarity solutions of the Navier-Stokes equations, to appear in Quader. di Mat.

[18] K. Ohkitani and H. Okamoto, Blow-up problems modeled from the strain-vorticity dynamics, Proceedings of "Tosio Kato's Method and Principles for Evolution Equations in Mathematical Physics" (Eds. H. Fujita, S. T. Kuroda and H. Okamoto), RIMS Kokyuroku 1234 (2001), pp. 240-250. Also at http://www.kurims.kyotou.ac.jp/ ${ }^{\circ}$ kamoto/paper/paper.html

[19] K. Ohkitani and J. D. Gibbon, Numerical study of singularity formation in a class of Euler and Navier-Stokes flows, Phys. Fluid, 12, 3181-3194 (2000).

[20] A. Pazy, Semigroups of Linear Operators and Applications to Partial Differential Equations, Springer Verlag (1983).

[21] H. Okamoto and J. Zhu, Some similarity solutions of the Navier-Stokes equations and related topics, Taiwanese J. Math., 4, 65-103 (2000). 